\title{
Poisson voting games under proportional rule
}

\author{
Francesco De Sinopoli ${ }^{1}$. Claudia Meroni ${ }^{2}([)$
}

Received: 10 December 2020 / Accepted: 20 September 2021 / Published online: 16 October 2021

(c) The Author(s) 2021

\begin{abstract}
We analyze strategic voting under proportional rule and two parties, embedding the basic spatial model into the Poisson framework of population uncertainty. We prove that there exists a unique Nash equilibrium. We show that it is characterized by a cutpoint in the policy space that is always located between the average of the two parties' positions and the median of the distribution of voters' types. We also show that, as the expected number of voters goes to infinity, the equilibrium converges to that of the case with deterministic population size.
\end{abstract}

\section{Introduction}

Citizens often act strategically as voters. Their expected behavior influences the competing parties' positioning choices, and their actions determine the electoral outcome and, consequently, the policy that is implemented according to the electoral rule in force. In parliamentary systems elections are usually conducted under proportional representation. In this case the policy outcome may be represented as a convex combination of the policies proposed by each party, with weights proportional to the number of votes that they obtain. Such an outcome function reflects the fact that every parliamentary member participates to the decisional process with the same power. In this paper we study voters' strategic behavior under this proportional rule, considering a unidimensional policy space and two parties.

In a mass election a voter is typically unaware of the exact number of other voters in the population but may have only some probabilistic information about it. To capture this kind of uncertainty we use the Poisson model introduced by Myerson (1998), in which the number of agents is not common knowledge but is a Poisson

Claudia Meroni

claudia.meroni@unimi.it

Francesco De Sinopoli

francesco.desinopoli@univr.it

1 Department of Economics, University of Verona, Via Cantarane 24, 37129 Verona, Italy

2 Department of Economics, Management and Quantitative Methods, University of Milan, Via Conservatorio 7, 20122 Milan, Italy 
distributed random variable. Being realistic to assume that the size of the electorate is random from the viewpoint of an individual, voting settings were the immediate application of Poisson games (Myerson 2000, 2002) and the Poisson framework has become the standard tool to model strategic voting in large elections (see, e.g., Feddersen and Pesendorfer 1999; Castanheira 2003; Krishna and Morgan 2011, 2012; Bouton and Castanheira 2012; Bouton 2013; Bouton and Gratton 2015; Herrera et al. 2014). ${ }^{1}$ In particular, Myerson (2002) analyzes strategic voting in singlewinner elections under different scoring rules, while Bouton (2013) and Bouton and Gratton (2015) study strategic voting in runoff elections. Herrera et al. (2014) examine turnout in proportional electoral systems with a costly rational voting model à la Ledyard (1984), where the strategic aspect regards the choice between voting and abstaining. So far, strategic voting under proportional representation focused on the choice of which party to vote for has not been explored in the context of population uncertainty. This work aims to fill such a lack in the literature.

We consider the basic spatial model in which the positions of two parties are exogenously given and voters have to choose which party to vote for. Voters can be of different types, each type being characterized by a bliss point in the policy space. We analyze the polar case in which the policy that is implemented after the voting stage is a weighted average of the two parties' positions, where weights are equal to the corresponding shares of votes. ${ }^{2}$ The further the implemented policy is from a type's bliss point, the worse off that type is.

In the context of deterministic population size, voters' strategic behavior in a pure proportional system with multiple parties has been analyzed in De Sinopoli and Iannantuoni (2007). They show that, as the number of voters grows to infinity, in equilibrium basically voters split in two and only the two extremist parties take votes. The policy outcome is precisely the "cutpoint outcome", that is, the outcome that is implemented when all the voters whose bliss points are on its left vote for the leftmost party and all the voters whose bliss points are on its right vote for the rightmost one. This result has been applied to other electoral systems exhibiting positive degrees of power sharing in Meroni (2017), where the limit equilibrium outcome is characterized for the two-party case. In the complete information model, the equilibrium and the equilibrium outcome are unique at the limit, when there is a continuum of voters. Before the limit, when the number of players is finite and individuals' incentives to act strategically can be fully investigated, there is a plethora of equilibria and the equilibrium outcome is indeterminate.

The population uncertainty model turns out to solve such an indeterminacy. In fact, we show that for every expected number of voters there exists a unique Nash

\footnotetext{
1 The convenient properties of Poisson games have been exploited to model also more general economic environments where a large number of actors are involved (see Satterthwaite and Shneyerov 2007; Makris 2008, 2009; Ritzberger 2009; Jehiel and Lamy 2015 among others).

2 This outcome is the utilitarian solution of a bargaining process among parliamentary members with a quadratic loss function if they are elected in proportion to their parties' vote shares. Hence, it can be reached as the result of a proto-coalition bargaining procedure as modelled in Baron and Diermeier (2001) when the status quo is quite negative. For a similar outcome function see, e.g., Ortuño-Ortín (1997) and Alesina and Rosenthal (2000).
} 
equilibrium. This is precisely due to the environmental equivalence property of Poisson games, for which every player's environment (including everyone in the game except herself) is the same and which does not hold if the population size is deterministic. ${ }^{3}$ The equilibrium is characterized by a "cutpoint type" so that voters divide exactly in two, those to the left of the cutpoint voting for the leftist party and those to its right voting for the rightist one. ${ }^{4}$ This is due to the fact that, in the Poisson environment, if a type prefers the rightist party to the leftist one for a given behavior of the population, then all the types located on her right will strictly do so. ${ }^{5}$ Moreover, if a type is indifferent between the two parties given a strategy of the others, she will strictly prefer the rightist party to the leftist one given another strategy such that the leftist party's vote share is larger. Then, the type indifferent against this second strategy will be on her left. A consequence is that the best response correspondence is a continuous decreasing function and this ensures the existence of a unique Nash equilibrium.

We examine the characteristics of the equilibrium with respect to the parameters of the model and show that the cutpoint always lies between the average of the two parties' positions and the median of the distribution of types. Furthermore, we study the behavior of the equilibrium cutpoint as the expected number of voters increases and show that it converges to the equilibrium cutpoint of the deterministic model with a continuum of voters.

Finally, we remove the hypothesis that parties' platforms are exogenously given and explore in an example their strategic decision of which position to take in the policy space when they are policy-motivated. Under such an assumption, the form of the outcome function implies that parties potentially face a trade-off. The more moderate is the policy that they choose, the larger is their share of votes but the less extreme is the position to which such a weight is assigned. We show that, if the expected number of voters is sufficiently large, parties choose extreme positions in equilibrium, in line with the results of the model with a continuum of strategic voters (Meroni 2017). However, this does not hold for every population size, depending on the distribution of voters' types. As the expected number of voters vanishes, for instance, parties' equilibrium positions may converge if the distribution is highly concentrated at a point in the policy space, in line with the results of the model with a continuum of sincere voters (see Saporiti 2014; Matakos et al. 2016). ${ }^{6}$

The paper is organized as follows. We describe the model in Sect. 2. In Sect. 3 we analyze its equilibrium and in Sect. 4 we examine how this varies as the population size increases. We present an example of parties' political competition in Sect. 5.

\footnotetext{
3 We refer to Myerson (1998) for the properties of Poisson games.

4 Diferently than under majoritarian rule, the cutpoint does not coincide with the average of the twoparties' positions, i.e. sincere voting is not necessarily an equilibrium. In fact, a voter may vote for thefurthest party if this moves the policy outcome closer to her bliss point.

5 We stress that this follows solely from environmental equivalence and does not hold with deterministicpopulation size. In particular, we note that population uncertainty unavoidably involves symmetricstrategies, but even focusing on symmetric equilibria does not guarantee uniqueness in the deterministicmodel.

6 Saporiti (2014) and Matakos et al. (2016) establish a positive relationship between the disproportionality of the electoral rule and the convergence of parties' positions in the case of, respectively, two parties with mixed motives and policy-motivated parties, when voters vote sincerely. Matakos et al. (2016) also show that polarization decreases in the density of moderate voters.
} 


\section{The model}

The policy space is represented by the closed interval $\mathbb{X}=[0,1]$. Two parties $L$ and $R$ are respectively located at positions $x_{L}, x_{R} \in \mathbb{X}$, with $x_{L}<x_{R}$. Every voter chooses which party to vote for, and the resulting electoral outcome determines the policy that is implemented according to the proportional rule. Precisely, let $v$ be the share of votes gained by party $L$. The policy outcome $x\left(v, x_{L}, x_{R}\right)$ is a weighted average of the two parties' positions where weights are given by the corresponding vote shares, i.e.

$$
x\left(v, x_{L}, x_{R}\right)=v x_{L}+(1-v) x_{R} .
$$

The number of voters is assumed to be a random variable drawn from a Poisson distribution with mean $n$. The probability that the actual number of voters is $k$, then, is equal to

$$
p(k \mid n)=\frac{e^{-n} n^{k}}{k !}
$$

Each voter has a type that determines her preferences over the possible outcomes. We identify the set of types with $\Theta=[0,1]$, where $\theta_{t} \in \Theta$ represents the bliss point of type $t$ voters, and we denote with $\theta$ the typical element of $\Theta$. Each voter's type is independently drawn from $\Theta$ according to some probability distribution $F$. That is, for every element $\theta \in \Theta$, the probability that a randomly sampled voter is of type $\theta_{t} \leq \theta$ is given by $F(\theta)$ and it is independent of the number and types of all the other voters. The decomposition property of the Poisson distribution implies that the number of voters with type smaller than or equal to $\theta$ is a Poisson random variable with parameter $n F(\theta)$. We assume that $F$ is continuous and strictly increasing.

We denote each voter's action set with $C=\{L, R\}$. An action profile $z=\left(z_{L}, z_{R}\right) \in \mathbb{Z}_{+}^{2}$ is a vector that specifies for each action $c \in C$ the number of voters who choose that action, $z_{c}$. The payoff to a voter of type $t$ who chooses action $c$ when the realization of the rest of the population's behavior is $z$ is a function of the distance between her bliss point and the induced policy. ${ }^{7}$ That is,

$$
\hat{u}_{t}(c, z)=f\left(\left|\theta_{t}-\hat{x}(c, z)\right|\right),
$$

where $\hat{x}(c, z)$ is the outcome that the voter induces choosing $c$ when $z=\left(z_{L}, z_{R}\right)$ is the action profile of the other voters, i.e

$$
\hat{x}(L, z)=\frac{z_{L}+1}{z_{L}+z_{R}+1} x_{L}+\frac{z_{R}}{z_{L}+z_{R}+1} x_{R}
$$

and

\footnotetext{
7 Differently from De Sinopoli and Iannantuoni (2007), where voters' preferences are only required to be single-peaked and representable by a continuously differentiable utility function, we also require them to be symmetric with respect to the bliss point. We need this assumption in the proof of Lemma 1.
} 


$$
\hat{x}(R, z)=\frac{z_{L}}{z_{L}+z_{R}+1} x_{L}+\frac{z_{R}+1}{z_{L}+z_{R}+1} x_{R} .
$$

Note that $\hat{x}(R, z)>\hat{x}(L, z)$ for every $z$. The function $f:[0,1] \rightarrow \mathbb{R}$ is assumed to be bounded, continuous, strictly decreasing, and strictly concave in its argument.

Given $x_{L}$ and $x_{R}$, with abuse of notation we denote with $x(z)$ the outcome that is implemented when $z$ is the entire population's realized behavior, i.e.

$$
x(z):=x\left(\frac{z_{L}}{z_{L}+z_{R}}, x_{L}, x_{R}\right) .
$$

Moreover, we denote with $u_{t}(x(z))$ the corresponding payoff to type $t$ voters. Clearly, $u_{t}(x(z))=\hat{u}_{t}\left(L, z^{\prime}\right)=\hat{u}_{t}\left(R, z^{\prime \prime}\right)$ where $z^{\prime}=\left(z_{L}-1, z_{R}\right)$ and $z^{\prime \prime}=\left(z_{L}, z_{R}-1\right)$. The function $u_{t}$ is strictly concave because $f$ is strictly concave, and it is assumed to be differentiable. ${ }^{8}$

A strategy function (or simply strategy) is a measurable function $\sigma: \Theta \rightarrow \Delta(C)$ which associates to each type a probability distribution on $C$, i.e. a mixed action. ${ }^{9}$ The mixed action employed by players of type $t$ is denoted $\sigma_{t}$. Strategy $\sigma$ induces the average behavior $\tau(\sigma) \in \Delta(C)$, which specifies for each action $c \in C$ the marginal probability that a randomly sampled voter chooses $c, \tau_{c}(\sigma)$, when players are expected to play according to $\sigma$. It is possible to see that $\tau_{c}(\sigma)$ is also the expected share of votes received by party $c .{ }^{10}$ The number of voters who choose $c$ is a Poisson random variable with mean $n \tau_{c}(\sigma)$ and it is independent of the number of voters who choose the other action.

Then, under the population's average behavior $\tau \in \Delta(C)$, the probability of the action profile $z \in Z(C)$ is given by

$$
P(z \mid \tau)=e^{-n} n^{z_{L}+z_{R}} \frac{\tau_{L}^{z_{L}} \tau_{R}^{z_{R}}}{z_{L} ! z_{R} !} .
$$

From the perspective of any player of any type, the number of other players (not including herself) who choose action $c$ is also a Poisson random variable with mean

\footnotetext{
8 This assumption is used in the proof of Theorem 2. We make it for simplicity to directly apply the mean value theorem, but it can be eliminated at the cost of using a more cumbersome version of that theorem.

${ }^{9}$ Differently from Myerson (2000), we do not use distributional strategies but we employ the equivalent definition of strategies in terms of measurable functions (see Milgrom and Weber 1985). We believe that the specification of the behavior of each single type is more appropriate in Poisson games, where agents are characterized only by their types and identities are unknown. Of course, all the strategy functions that differ only in a finite subset of $\Theta$ are outcome equivalent, as such a subset has measure zero being the function $F$ continuous. Hence, a distributional strategy identifies a class of equivalence of our strategy functions.

${ }^{10}$ When the realized number of voters is zero, party $c$ 's share of votes is not defined. For every average behavior $\tau \in \Delta(C)$ we assume it to be equal to $\tau_{c}$, as it is for every positive realization of the population. As a matter of fact, the expected share of votes of party $c$ given $\tau$ is given by $\sum_{m=0}^{\infty} p(m \mid n) \sum_{z_{c}=0}^{m}\left(\begin{array}{c}m \\ z_{c}\end{array}\right) \tau_{c} z_{c}\left(1-\tau_{c}\right)^{m-z_{c}} \frac{z_{c}}{m}=\sum_{m=0}^{\infty} p(m \mid n) \frac{m \tau_{c}}{m}$. Clearly, this convenient simplifying assumption does not affect any result obtained for $n$ sufficiently large.
} 
$n \tau_{c}$ due to the environmental equivalence property of Poisson games. Thus, the expected payoff to a type $t$ voter who votes for $c$ when the other voters vote according to $\tau$ is given by

$$
U_{t}(c, \tau)=\sum_{z \in Z(C)} P(z \mid \tau) u_{t}(c, z)
$$

Following standard terminology, we say that action $c$ is a pure best response for type $t$ voters against the population's behavior $\tau$ if $U_{t}(c, \tau) \geq U_{t}\left(c^{\prime}, \tau\right)$ for $c^{\prime} \neq c$. We denote the set of such actions $\operatorname{PBR}_{t}(\tau)$. The set of type $t$ voters' best responses against $\tau$ is $\mathrm{BR}_{t}(\tau)=\Delta\left(\operatorname{PBR}_{t}(\tau)\right)$, and $\mathrm{BR}(\tau)$ is the collection of strategy functions such that $\sigma_{t} \in \mathrm{BR}_{t}(\tau)$ for every $t$.

Definition 1 The strategy function $\sigma$ is a Nash equilibrium if $\sigma \in \operatorname{BR}(\tau(\sigma))$.

\section{Equilibrium analysis}

In this section we prove that the model has a unique Nash equilibrium. We show that in equilibrium voters split in two, those to the left of a "cutpoint type" voting for party $L$ and those to its right voting for party $R$.

Formally, we say that strategy $\sigma$ is a cutpoint strategy if there is a value $\theta \in \Theta$ such that $\sigma_{t}(L)=1$ if $\theta_{t}<\theta$ and $\sigma_{t}(R)=1$ if $\theta_{t}>\theta$. That is, all the types to the left of type $\theta$ vote for party $L$ and all the types to the right of $\theta$ vote for party $R$. We will usually refer to a cutpoint strategy with the correspondent cutpoint. Note that a given $\theta$ identifies an infinite number of strategy functions that differ only at the point $\theta_{t}=\theta$, which are equivalent given the continuity of the function $F$.

We begin proving that, for any average behavior $\tau$, the best response against $\tau$ is a cutpoint strategy and is unique, i.e. the best response correspondence is a function. In fact, the assumptions on the utility function imply that, given an average population behavior, if a type prefers to vote for party $R$ than for party $L$ then all the types on her right will strictly do so. ${ }^{11}$ Not only this holds for every average behavior, but it holds for every realization of the population behavior. We prove first such a stronger result.

Lemma 1 For every $z \in Z(C)$ and $\theta_{t^{\prime}}>\theta_{t}$, if type t prefers $R$ to $L$ given $z$ then type $t^{\prime}$ strictly prefers $R$ to $L$ given $z$.

Proof Fix $z \in Z(C)$ and let $\Delta_{t}=\hat{u}_{t}(R, z)-\hat{u}_{t}(L, z)$ for every $t$. Recall that $\hat{x}(R, z)>\hat{x}(L, z)$ and consider $\theta_{t^{\prime}}>\theta_{t}$. There are six possible ways in which the points $\hat{x}(R, z), \hat{x}(L, z), \theta_{t^{\prime}}$, and $\theta_{t}$ are ordered in the interval $[0,1]$. For each case, the strict decreasingness and strict concavity of the function $f$ imply the following:

11 When strictness is not specified, preference is meant to be weak. 
1. if $\hat{x}(L, z)<\hat{x}(R, z) \leq \theta_{t}<\theta_{t^{\prime}}$ then $0<\Delta_{t}<\Delta_{t^{\prime}}$;

2. if $\theta_{t}<\theta_{t^{\prime}} \leq \hat{x}(L, z)<\hat{x}(R, z)$ then $\Delta_{t}<\Delta_{t^{\prime}}<0$;

3. if $\theta_{t} \leq \hat{x}(L, z)<\hat{x}(R, z) \leq \theta_{t^{\prime}}$ then $\Delta_{t}<0<\Delta_{t^{\prime}}$;

4. if $\hat{x}(L, z) \leq \theta_{t}<\theta_{t^{\prime}} \leq \hat{x}(R, z)$ then either $\Delta_{t}<0<\Delta_{t^{\prime}}$ or $0 \leq \Delta_{t}<\Delta_{t^{\prime}}$ or $\Delta_{t}<\Delta_{t^{\prime}} \leq 0$

5. if $\hat{x}(L, z) \leq \theta_{t} \leq \hat{x}(R, z) \leq \theta_{t^{\prime}}$ then either $\Delta_{t} \leq 0<\Delta_{t^{\prime}}$ or $0<\Delta_{t}<\Delta_{t^{\prime}}$;

6. if $\theta_{t} \leq \hat{x}(L, z) \leq \theta_{t^{\prime}} \leq \hat{x}(R, z)$ then either $\Delta_{t}<0 \leq \Delta_{t^{\prime}}$ or $\Delta_{t}<\Delta_{t^{\prime}}<0$.

In every case we have $\Delta_{t^{\prime}}>\Delta_{t}$, that is, $\hat{u}_{t^{\prime}}(R, z)-\hat{u}_{t^{\prime}}(L, z)>\hat{u}_{t}(R, z)-\hat{u}_{t}(L, z)$. It follows that if $\hat{u}_{t}(R, z) \geq \hat{u}_{t}(L, z)$ then $\hat{u}_{t^{\prime}}(R, z)>\hat{u}_{t^{\prime}}(L, z)$.

Recall that the environmental equivalence property of Poisson games implies that, for every average behavior $\tau$ and action profile $z$, each type attaches the same probability $P(z \mid \tau)$ to $z$. Thus, it follows readily from Lemma 1 that, for every $\tau$ and every $\theta_{t^{\prime}}>\theta_{t}$, if $U_{t}(R, \tau) \geq U_{t}(L, \tau)$ then $U_{t^{\prime}}(R, \tau)>U_{t^{\prime}}(L, \tau)$.

Lemma 2 For every $\tau \in \Delta(C)$ and $\theta_{t^{\prime}}>\theta_{t}$, if type t prefers $R$ to $L$ given $\tau$ then type $t^{\prime}$ strictly prefers $R$ to L given $\tau$.

A direct consequence of Lemma 2 is that $\mathrm{BR}(\tau)$ is a cutpoint strategy. In fact the previous result implies that, for every given $\tau$, there must be a cutpoint such that all the voters to its right strictly prefer $R$ over $L$ while all the voters to its left strictly prefer $L$ over $R .^{12}$ The type whose bliss point is exactly the cutpoint is indifferent between $L$ and $R$ given $\tau$. In addition, the above lemma excludes that there could be more than one indifferent type. Thus, for every $\tau$, the cutpoint that characterizes $\mathrm{BR}(\tau)$ is unique.

Proposition 1 For every $\tau \in \Delta(C), \mathrm{BR}(\tau)$ is a cutpoint strategy and is unique.

It follows that every equilibrium of the model is a cutpoint strategy and is therefore identified by a point in $\Theta$. We are going to prove the existence of a unique Nash equilibrium exploiting this fact, which allows to consider the restriction of the best response correspondence to cutpoint strategies. That is, we consider the function $\operatorname{BR}(\theta):[0,1] \rightarrow[0,1]$, and we show that it is continuous and decreasing.

Theorem 1 There exists a unique Nash equilibrium.

Proof Given a cutpoint strategy $\theta$, note that the probability that a randomly sampled voter votes for party $L$ (resp. $R$ ) is given by $\tau_{L}(\theta)=F(\theta)\left(\right.$ resp. $\tau_{R}(\theta)=1-F(\theta)$ ). By Proposition $1, \mathrm{BR}(\theta)$ is characterized by the point $\theta_{t} \in \Theta$ such that $U_{t}(R, \tau(\theta))-U_{t}(L, \tau(\theta))=0$, that is,

\footnotetext{
12 Notice that, given any population behavior, the type located at 0 strictly prefers $L$ over $R$, while the type located at 1 strictly prefers $R$ over $L$. Hence, given the assumptions on $F$, the indifferent type always exists and is strictly between 0 and 1 .
} 


$$
\sum_{z \in Z(C)} e^{-n} n^{z_{L}+z_{R}} \frac{F(\theta)^{z_{L}}[1-F(\theta)]^{z_{R}}}{z_{L} ! z_{R} !}\left[\hat{u}_{t}(R, z)-\hat{u}_{t}(L, z)\right]=0 .
$$

It is easy to see that $\operatorname{BR}(\theta)$ is continuous in $\theta$ given that $F(\theta)$ is continuous in $\theta$. Therefore, the Brouwer fixed point theorem readily implies that $\operatorname{BR}(\theta)$ has at least one fixed point, that is, an equilibrium exists. ${ }^{13}$

We can now prove that $\operatorname{BR}(\theta)$ is decreasing in $\theta$. In fact we can show that, given two cutpoint strategies $\theta$ and $\theta^{\prime}$ such that $\theta^{\prime}>\theta$, if $U_{t}(R, \tau(\theta))=U_{t}(L, \tau(\theta))$ then $U_{t}\left(R, \tau\left(\theta^{\prime}\right)\right)>U_{t}\left(L, \tau\left(\theta^{\prime}\right)\right)$. That is, the type $t$ who is indifferent between $R$ and $L$ given $\theta$ strictly prefers $R$ to $L$ given $\theta^{\prime}$. By Lemma 2, then, the indifferent type against $\theta^{\prime}$ will be on the left of type $t$, i.e. $B R\left(\theta^{\prime}\right)<B R(\theta)$.

First note that, for every $t$ and every couple of realizations $z$ and $z^{\prime}$ such that $z=\left(z_{L}, z_{R}\right)$ and $z^{\prime}=\left(z_{L}+1, z_{R}-1\right)$, we have

$$
\hat{u}_{t}\left(R, z^{\prime}\right)-\hat{u}_{t}\left(L, z^{\prime}\right)>\hat{u}_{t}(R, z)-\hat{u}_{t}(L, z) .
$$

Indeed, since $\hat{u}_{t}\left(R, z^{\prime}\right)=\hat{u}_{t}(L, z)=u_{t}\left(x\left(z_{L}+1, z_{R}\right)\right)$, the above condition is equivalent to

$$
u_{t}\left(x\left(z_{L}+1, z_{R}\right)\right)>\frac{1}{2} u_{t}\left(x\left(z_{L}+2, z_{R}-1\right)\right)+\frac{1}{2} u_{t}\left(x\left(z_{L}, z_{R}+1\right)\right) .
$$

Given that

$$
x\left(z_{L}+1, z_{R}\right)=\frac{1}{2} x\left(z_{L}+2, z_{R}-1\right)+\frac{1}{2} x\left(z_{L}, z_{R}+1\right),
$$

condition (1) follows directly from the strict concavity of the utility function.

Then, note that for every $t$ and $\theta \in \Theta$ we can write

$$
\begin{aligned}
& U_{t}(R, \tau(\theta))-U_{t}(L, \tau(\theta))=\sum_{m=0}^{\infty} \frac{e^{-n} n^{m}}{m !} \sum_{z_{L}=0}^{m}\left(\begin{array}{c}
m \\
z_{L}
\end{array}\right) F(\theta)^{z_{L}} \\
& \quad \times[1-F(\theta)]^{m-z_{L}}\left[\hat{u}_{t}\left(R,\left(z_{L}, m-z_{L}\right)\right)-\hat{u}_{t}\left(L,\left(z_{L}, m-z_{L}\right)\right)\right] .
\end{aligned}
$$

Fix the realization of the population $m>0$. For every $t$ and $z_{L} \leq m$, let

$$
\Delta_{t}\left(z_{L}\right):=\hat{u}_{t}\left(R,\left(z_{L}, m-z_{L}\right)\right)-\hat{u}_{t}\left(L,\left(z_{L}, m-z_{L}\right)\right) .
$$

Given the result above, we have $\Delta_{t}\left(z_{L}\right)>\Delta_{t}\left(z_{L}-1\right)$ for every $0<z_{L} \leq m$. Let also

$$
B\left(z_{L} \mid \theta\right):=\left(\begin{array}{c}
m \\
z_{L}
\end{array}\right) F(\theta)^{z_{L}}[1-F(\theta)]^{m-z_{L}}
$$

denote the probability that the number of voters choosing $L$ is equal to $z_{L}$ given that the population size is $m$ and given the cutpoint strategy $\theta \in \Theta$, and consider $\theta^{\prime}>\theta$.

\footnotetext{
13 See Myerson (2000) for existence of equilibria in Poisson games with compact type sets and finite action sets.
} 
The binomial distribution with parameters $m$ and $F\left(\theta^{\prime}\right)$ first order stochastically dominates the one with parameters $m$ and $F(\theta)$, that is,

$$
\sum_{z_{L}=0}^{\bar{z}} B\left(z_{L} \mid \theta^{\prime}\right)<\sum_{z_{L}=0}^{\bar{z}} B\left(z_{L} \mid \theta\right)
$$

for every $\bar{z}<m$. Therefore, for every $t$, we have

$$
\begin{array}{rl}
\sum_{z_{L}=0}^{m} & B\left(z_{L} \mid \theta\right) \Delta_{t}\left(z_{L}\right) \\
< & B\left(0 \mid \theta^{\prime}\right) \Delta_{t}(0)+\left[B(1 \mid \theta)+B(0 \mid \theta)-B\left(0 \mid \theta^{\prime}\right)\right] \Delta_{t}(1)+\sum_{z_{L}=2}^{m} B\left(z_{L} \mid \theta\right) \Delta_{t}\left(z_{L}\right) \\
& <B\left(0 \mid \theta^{\prime}\right) \Delta_{t}(0)+B\left(1 \mid \theta^{\prime}\right) \Delta_{t}(1)+\left[B(2 \mid \theta)+B(0 \mid \theta)-B\left(0 \mid \theta^{\prime}\right)+B(1 \mid \theta)\right. \\
& \left.-B\left(1 \mid \theta^{\prime}\right)\right] \Delta_{t}(2)+\sum_{z_{L}=3}^{m} B\left(z_{L} \mid \theta\right) \Delta_{t}\left(z_{L}\right)<\cdots \\
& <\sum_{z_{L}=0}^{m-1} B\left(z_{L} \mid \theta^{\prime}\right) \Delta_{t}\left(z_{L}\right)+\left[B(m \mid \theta)+\sum_{z_{L}=0}^{m-1} B\left(z_{L} \mid \theta\right)-\sum_{z_{L}=0}^{m-1} B\left(z_{L} \mid \theta^{\prime}\right)\right] \Delta_{t}(m) \\
= & \sum_{z_{L}=0}^{m} B\left(z_{L} \mid \theta^{\prime}\right) \Delta_{t}\left(z_{L}\right)+\left[\sum_{z_{L}=0}^{m} B\left(z_{L} \mid \theta\right)-\sum_{z_{L}=0}^{m} B\left(z_{L} \mid \theta^{\prime}\right)\right] \Delta_{t}(m) \\
= & \sum_{z_{L}=0}^{m} B\left(z_{L} \mid \theta^{\prime}\right) \Delta_{t}\left(z_{L}\right),
\end{array}
$$

where the first inequality derives from $B(0 \mid \theta)-B\left(0 \mid \theta^{\prime}\right)>0$ and $\Delta_{t}(1)>\Delta_{t}(0)$, the second inequality derives from $B(0 \mid \theta)+B(1 \mid \theta)-B\left(0 \mid \theta^{\prime}\right)-B\left(1 \mid \theta^{\prime}\right)>0$ and $\Delta_{t}(2)>\Delta_{t}(1)$, and so on until the last inequality, which derives from $\sum_{z_{L}=0}^{m-1} B\left(z_{L} \mid \theta\right)-\sum_{z_{L}=0}^{m-1} B\left(z_{L} \mid \theta^{\prime}\right)>0$ and $\Delta_{t}(m)>\Delta_{t}(m-1){ }^{14}$ As this holds for every realization $m>0$, we can conclude that

$$
U_{t}(R, \tau(\theta))-U_{t}(L, \tau(\theta))<U_{t}\left(R, \tau\left(\theta^{\prime}\right)\right)-U_{t}\left(L, \tau\left(\theta^{\prime}\right)\right)
$$

for every $t .^{15}$

Thus, if $U_{t}(R, \tau(\theta))=U_{t}(L, \tau(\theta)) \quad$ then $\quad U_{t}\left(R, \tau\left(\theta^{\prime}\right)\right)>U_{t}\left(L, \tau\left(\theta^{\prime}\right)\right)$, so $B R\left(\theta^{\prime}\right)<B R(\theta)$. Since the best response function is (strictly) decreasing, the Nash equilibrium is unique.

We conclude this section showing some characteristics of the Nash equilibrium relatively to the parameters of the model. In particular, we can prove that the equilibrium cutpoint is always located between the average of the two parties' positions and the median of the distribution of voters' types. To this end, let $\theta^{*}$ be the equilibrium cutpoint, let $\bar{\theta}=\frac{x_{L}+x_{R}}{2}$ and $F\left(\theta_{m}\right)=\frac{1}{2}$.

\footnotetext{
14 Of course, if $m=1$ we have just one inequality, if $m=2$ we have two inequalities, etc.

15 Note that for $m=0$ we have $B(0 \mid \theta)=B\left(0 \mid \theta^{\prime}\right)=1$.
} 
Proposition 2 If $\theta_{m}>\bar{\theta}$ then $\bar{\theta}<\theta^{*}<\theta_{m}$, while if $\theta_{m}<\bar{\theta}$ then $\theta_{m}<\theta^{*}<\bar{\theta}$.

Proof Consider the cutpoint strategy $\theta_{m}$, which induces the average behavior $\tau$ such that $\tau_{L}\left(\theta_{m}\right)=\tau_{R}\left(\theta_{m}\right)=\frac{1}{2}$. Any two symmetric realizations, i.e. any two realizations $z$ and $z^{\prime}$ such that $z=\left(z_{1}, z_{2}\right)$ and $z^{\prime}=\left(z_{2}, z_{1}\right)$, have the same probability given $\theta_{m}$. Let $t$ be the type whose bliss point is $\bar{\theta}$. For every realization $z$ such that $z_{L}=z_{R}$ we have $\hat{u}_{t}(R, z)=\hat{u}_{t}(L, z)$, while for every symmetric realizations $z$ and $z^{\prime}$ we have $\hat{u}_{t}(R, z)-\hat{u}_{t}(L, z)=\hat{u}_{t}\left(L, z^{\prime}\right)-\hat{u}_{t}\left(R, z^{\prime}\right)$. It follows that type $t$ is indifferent between choosing $L$ and choosing $R$ given $\theta_{m}$, that is, $B R\left(\theta_{m}\right)=\bar{\theta}$. Since the best response function is continuous and strictly decreasing, both when $\theta_{m}>\bar{\theta}$ and when $\theta_{m}<\bar{\theta}$ its fixed point lies between these two values. Of course, if $\theta_{m}=\bar{\theta}$ then $\theta^{*}=\theta_{m}=\bar{\theta}$.

The results presented in this section hold for every value of the expected number of voters $n$. In the next section we study the behavior of the Nash equilibrium as the expected population size increases.

\section{Large electorate}

If we fix the parties' platforms $x_{L}$ and $x_{R}$ and the distribution of voters' types $F$, the Nash equilibrium is a function of the expected number of voters $n, \theta^{*}(n)$. In this section we study the behavior of this function as the electorate becomes large. In particular we show that, as $n$ goes to infinity, the equilibrium cutpoint converges to that of the deterministic model with a continuum of voters studied in De Sinopoli and Iannantuoni (2007).

To this end, let the cutpoint outcome $\hat{\theta}$ be the unique policy outcome implemented when all the types to its left vote for party $L$ and all the types to its right vote for party $R$, i.e. the unique solution to

$$
\hat{\theta}=F(\hat{\theta}) x_{L}+[1-F(\hat{\theta})] x_{R} .
$$

De Sinopoli and Iannantuoni (2007) prove that $\hat{\theta}$ is the unique equilibrium of the model with deterministic population size in which there is a continuum of voters, when this is seen as the limit of a sequence of finite games. ${ }^{16} \mathrm{We}$ are going to prove that $\hat{\theta}$ is the limit point of the equilibrium cutpoint of the Poisson model as the expected number of voters grows to infinity.

\footnotetext{
16 We refer to the "unique equilibrium" of a game with a continuum of players with slight abuse of terminology. Loosely speaking, De Sinopoli and Iannantuoni (2007) prove that, given a converging sequence of finite games, each game in the sequence has two particular voters; the rightmost one such that, in every equilibrium, she and all the voters to her left vote for party $L$ and the leftmost one such that, in every equilibrium, she and all the voters to her right vote for party $R$. The two corresponding sequences of these voters' bliss points converge to the same limit point, which is precisely the cutpoint outcome associated to the distribution function of voters' bliss points of the limit game (see Section 3.3 in De Sinopoli and Iannantuoni 2007).
} 
First, we present some results that derive from the properties of the Poisson distribution. We begin reminding that there exist exponentially decreasing bounds for the tail probabilities of a Poisson random variable. ${ }^{17}$ This directly implies that, in a Poisson game, the probability of the population realizations that are smaller than a given value becomes smaller and smaller as $n$ increases.

Lemma 3 For every $\delta>0$ and $\bar{m}>0$ there exists a value $\bar{n} \in \mathbb{R}$ such that, for every $n \geq \bar{n}, \sum_{m=0}^{\bar{m}} p(m \mid n)<\delta$.

The second result regards the parties' shares of votes. Given the average behavior $\tau$ and fixed the realization of the population $m$, the vote share of party $L$ is a random variable that takes value $\frac{z_{L}}{m}$ with probability $\left(\begin{array}{c}m \\ z_{L}\end{array}\right) \tau_{L}^{z_{L}}\left(1-\tau_{L}\right)^{m-z_{L}}$ (similarly for the vote share of party $R$ ). Let us denote such a variable $\tilde{s}(m)$. Note that

$$
\tilde{s}(m)=\sum_{i=1}^{m} \frac{\tilde{\omega}_{i}}{m},
$$

where $\tilde{\omega}_{i}$ is a player's vote for party $L$ given $\tau$, i.e. the random variable that takes value 1 with probability $\tau_{L}$ and 0 with probability $1-\tau_{L}$. Let $\operatorname{Pr}\left(\left|\tilde{s}(m)-\tau_{L}\right|>\varepsilon\right)$ denote the probability that $\tilde{s}(m)$ is further than $\varepsilon$ from its expected value $\tau_{L}$. The next result follows directly from the weak law of large numbers, which states that the sample mean converges in probability to the expected value.

Lemma 4 For every $\delta>0$ and $\varepsilon>0$ there exists a value $\bar{m} \in \mathbb{R}$ such that, for every $m \geq \bar{m}, \operatorname{Pr}\left(\left|\tilde{s}(m)-\tau_{L}\right|>\varepsilon\right)<\delta$.

We can use Lemmas 3 and 4 to prove that the sample mean converges in probability to the expected value also when the size of the population is a Poisson random variable. This gives a clear intuition of the fact that the policy outcome, which is a continuous function of the shares of votes, remains sufficiently close to its expected value with probability sufficiently high if $n$ is large enough. Such a fact is at the basis of the result in Theorem $2 .^{18}$

Lemma 5 For every $\delta>0$ and $\varepsilon>0$ there exists a value $\bar{n} \in \mathbb{R}$ such that, for every $n \geq \bar{n}$

$$
\sum_{m=0}^{\infty} p(m \mid n)[\operatorname{Pr}(|\tilde{s}(m)-\tau(L)|>\varepsilon)]<\delta .
$$

\footnotetext{
17 Given a Poisson random variable with parameter $n$, the Chernoff bound implies that $\sum_{k=0}^{h} p(k \mid n) \leq e^{-n}(e n)^{h} / h^{h}$ for $h<n$ and $1-\sum_{k=0}^{h-1} p(k \mid n) \leq e^{-n}(e n)^{h} / h^{h}$ for $h>n$.
}

18 We will also use it in the next section to prove Proposition 3. 
Proof Given $\delta>0$ and $\varepsilon>0$, let $\delta_{1}=\delta_{2}=\delta / 2$. By Lemma 4, there exists a value $\bar{m}$ such that, for every $m \geq \bar{m}, \operatorname{Pr}(|\tilde{s}(m)-\tau(L)|>\varepsilon)<\delta_{1}$. Given $\bar{m}$, then, there exists by Lemma 3 a value $\bar{n}$ such that, for every $n \geq \bar{n}, \sum_{m=0}^{\bar{m}} p(m \mid n)<\delta_{2}$. Therefore, for $n \geq \bar{n}$ we have

$$
\begin{aligned}
& \sum_{m=0}^{\infty} p(m \mid n)[\operatorname{Pr}(|\tilde{s}(m)-\tau(L)|>\varepsilon)] \\
& \quad=\sum_{m=0}^{\bar{m}} p(m \mid n)[\operatorname{Pr}(|\tilde{s}(m)-\tau(L)|>\varepsilon)]+\sum_{m=\bar{m}+1}^{\infty} p(m \mid n)[\operatorname{Pr}(|\tilde{s}(m)-\tau(L)|>\varepsilon)] \\
& \quad<\sum_{m=0}^{\bar{m}} p(m \mid n)+\sum_{m=\bar{m}+1}^{\infty} p(m \mid n) \delta_{1}<\delta_{2}+\delta_{1}=\delta .
\end{aligned}
$$

Given the average behavior $\tau \in \Delta(C)$, let $X(\tau)$ denote the expected outcome, i.e. $^{19}$

$$
X(\tau)=\tau_{L} x_{L}+\left(1-\tau_{L}\right) x_{R} .
$$

We can now prove that, for $n$ sufficiently large, the equilibrium cutpoint lies in a neighborhood of the expected equilibrium outcome which shrinks to zero as $n$ goes to infinity. Since the equilibrium cutpoint and the expected equilibrium outcome are always located on opposite sides relative to the cutpoint outcome, it follows that the equilibrium cutpoint lies in a neighborhood of the cutpoint outcome of the same length. Thence, the limit point of the equilibrium cutpoint $\theta^{*}(n)$ as $n$ goes to infinity is the cutpoint outcome $\hat{\theta}$.

Let $\sigma^{*}(n)$ be the Nash equilibrium characterized by the cutpoint $\theta^{*}(n)$, and let $\tau^{*}(n)=\tau\left(\sigma^{*}(n)\right)$.

Theorem 2 For every $\delta>0$ there exists a value $\bar{n} \in \mathbb{R}$ such that, for every $n \geq \bar{n}$, $\left|\theta^{*}(n)-X\left(\tau^{*}(n)\right)\right|<\delta$. Furthermore, $\left|\hat{\theta}-\theta^{*}(n)\right|<\delta$ and $\left|\hat{\theta}-X\left(\tau^{*}(n)\right)\right|<\delta$.

\section{Proof See Appendix A.}

Remark 1 From the results of this section it follows that the model with large electorate is completely analogous to the limit model with deterministic population size, as the number of voters goes to infinity. In particular, if we extend our analysis to the case in which there are more than two parties as in De Sinopoli and Iannantuoni (2007), we have that as $n$ goes to infinity in equilibrium only the two most extreme parties take votes. With deterministic population size, indeed, in equilibrium all the voters except those in a neighborhood of the equilibrium outcome choose the extremist parties, in order to drag the policy outcome towards their bliss point. That neighborhood reduces as the number of voters increases. An equivalent result is true

19 Recall the assumption in footnote 5 for the event in which no player realizes. 
in the Poisson environment where, as the expected population size increases, the probability of low realizations goes rapidly to zero. Before the limit there is multiplicity of equilibria notwithstanding environmental equivalence because, intuitively, central voters can "coordinate" on different behaviors. ${ }^{20}$

\section{Parties' political competition: an example}

In this section we remove the hypothesis that parties' platforms are exogenous and discuss an example of the political competition that happens before the voting stage; that is, parties' strategic decision of which position to take in the policy space. In particular, we assume that parties $L$ and $R$ are purely policy-motivated with the same preferences as voters, and are characterized respectively by ideal policies 0 and 1 for simplicity. We let $x_{L}, x_{R} \in \mathbb{X}$ denote the positions that they choose, removing the assumption $x_{L}<x_{R}{ }^{21}$ We assume that the distribution of voters' types $F$ is also differentiable.

In the deterministic model with a continuum of strategic voters, Meroni (2017) shows that two policy-motivated parties choose extreme positions in equilibrium, in contrast with the results of the model in which voters vote sincerely (Saporiti 2014; Matakos et al. 2016). The same result is true in the model where the number of voters is Poisson distributed when its expected value is sufficiently large. In fact we can show that, loosely speaking, by changing the chosen platform a party moves the implemented policy in the same direction of the change. Hence, in equilibrium, the centripetal force due to the incentive to obtain a larger vote share is dominated by the centrifugal force due to the incentive to assign that weight to a more extreme platform, and parties' positions diverge.

Proposition 3 There exists a value $\bar{n} \in \mathbb{R}$ such that, for every $n \geq \bar{n}$, the unique Nash equilibrium is $\left(x_{L}, x_{R}\right)=(0,1)$.

\section{Proof See Appendix B.}

Divergence of parties' positions does not extend to every expected number of voters, depending on the distribution of voters' types $F$. To see this note that if $n$ is sufficiently small then the equilibrium of the voting game remains sufficiently close to the average of parties' positions. ${ }^{22}$ Differently from the cutpoint outcome, that value does not depend on the distribution function $F$, so in this case parties' positions can converge for some specifications of that function.

\footnotetext{
${ }^{20}$ We thank an anonymous referee for a comment that led to this remark.

21 If $x_{L}=x_{R}$, any strategy function is a Nash equilibrium of the voting game, but the equilibrium outcome coincides always with the two proposed policies.

22 As the expected number of voters vanishes, the probability for a voter of being the only player in the game converges to one. It readily follows that the equilibrium cutpoint converges to the average of the two parties' positions, that is, voters vote sincerely.
} 
Consider for example a distribution $F$ that is highly concentrated in a sufficiently small neighborhood of $\theta=\frac{1}{3}$ and suppose that parties choose positions $x_{L}=0$ and $x_{R}=1$. If $n$ is close to zero, the equilibrium cutpoint of the voting game is close to $\bar{\theta}=\frac{1}{2}$. Therefore, the expected vote share of party $L$ is close to one, and with probability close to one the outcome is close to 0 . It follows that party $R$ has now the incentive to deviate to a more moderate position to increase her expected vote share and bring the outcome closer to $1 .^{23}$

Remark 2 The assumption of extreme parties' ideal policies is needed only to simplify the proof of the above divergence result. In the proof it is shown that at the limit the behavior of voters and the implemented outcome converge to those of the model with deterministic population size (see Appendix B). That proof can be extended to general ideal policies for parties at the cost of a much heavier notation and more cumbersome steps. In particular, in line with Meroni (2017), if $n$ is sufficiently large then in the unique equilibrium at least one party chooses an extreme position while the other party chooses a position that is always more extreme than its ideal policy. In fact, if the cutpoint $\hat{\theta}_{0,1}$ when parties' chosen platforms are 0 and 1 lies between the two parties ideal policies $\theta_{L}$ and $\theta_{R}$, then in equilibrium parties choose positions 0 and 1 . On the other hand, if $\theta_{L}<\theta_{R}<\hat{\theta}_{0,1}$ then party $L$ chooses position 0 and party $R$ chooses a position between $\theta_{R}$ and 1 , while if $\hat{\theta}_{0,1}<\theta_{L}<\theta_{R}$ then party $L$ chooses a position between 0 and $\theta_{L}$ and party $R$ chooses position 1 .

\section{Appendix A: Proof of Theorem 2}

Theorem 2 For every $\delta>0$ there exists a value $\bar{n} \in \mathbb{R}$ such that, for every $n \geq \bar{n}$, $\left|\theta^{*}(n)-X\left(\tau^{*}(n)\right)\right|<\delta$. Furthermore, $\left|\hat{\theta}-\theta^{*}(n)\right|<\delta$ and $\left|\hat{\theta}-X\left(\tau^{*}(n)\right)\right|<\delta$.

Proof We start showing that, for every $\delta>0$, there exists a value $\bar{n}_{L} \in \mathbb{R}$ such that, for every $n \geq \bar{n}_{L}$, if $\theta_{t} \leq X\left(\tau^{*}(n)\right)-\delta$ then $\sigma_{t}^{*}(n)(L)=1$. This, together with an analogous and symmetric result for $\theta_{t} \geq X\left(\tau^{*}(n)\right)-\delta$, implies the first statement of the theorem.

Fix $\delta$, take a type $t$ such that $\theta_{t} \leq X\left(\tau^{*}(n)\right)-\delta$ and consider the difference of utility that she gets choosing $R$ instead of $L$ given the equilibrium behavior $\tau^{*}(n)$. We have

$$
\begin{aligned}
& U_{t}\left(R, \tau^{*}(n)\right)-U_{t}\left(L, \tau^{*}(n)\right) \\
& \quad=\sum_{m=0}^{\infty} p(m \mid n) \sum_{z_{L}=0}^{m}\left(\begin{array}{c}
m \\
z_{L}
\end{array}\right) \tau_{L}^{*}(n)^{z_{L}}\left(1-\tau_{L}^{*}(n)\right)^{m-z_{L}}\left[u_{t}\left(\hat{x}\left(R, z_{L}, m-z_{L}\right)\right)-u_{t}\left(\hat{x}\left(L, z_{L}, m-z_{L}\right)\right)\right] \\
& \quad=\sum_{m=0}^{\infty} p(m \mid n) \sum_{z_{L}=0}^{m} \operatorname{Pr}\left(\tilde{s}(m)=\frac{z_{L}}{m} \mid \tau^{*}(n)\right)\left[u_{t}\left(\hat{x}\left(R, z_{L}, m-z_{L}\right)\right)-u_{t}\left(\hat{x}\left(R, z_{L}, m-z_{L}\right)-\frac{x_{R}-x_{L}}{m+1}\right)\right]
\end{aligned}
$$

\footnotetext{
${ }^{23}$ The same result holds also if we assume that the outcome when no player realizes is an exogenous status quo.
} 
as $\hat{x}\left(R, z_{L}, m-z_{L}\right)-\hat{x}\left(L, z_{L}, m-z_{L}\right)=\frac{x_{R}-x_{L}}{m+1}$, where $\operatorname{Pr}\left(\tilde{s}(m)=\frac{z_{L}}{m} \mid \tau^{*}(n)\right)$ denotes the probability that the vote share of party $L$ is equal to $\frac{z_{L}}{m}$ given $\tau^{*}(n)$.

Since the utility function $u_{t}$ is continuous on $[0,1]$ and differentiable on $(0,1)$, the mean value theorem implies that for each $m$ and $z_{L}$ there exists a value

$$
\bar{x} \in\left[\hat{x}\left(R, z_{L}, m-z_{L}\right)-\frac{x_{R}-x_{L}}{m+1}, \hat{x}\left(R, z_{L}, m-z_{L}\right)\right]
$$

such that

$$
u_{t}\left(\hat{x}\left(R, z_{L}, m-z_{L}\right)\right)-u_{t}\left(\hat{x}\left(R, z_{L}, m-z_{L}\right)-\frac{x_{R}-x_{L}}{m+1}\right)=\left.\frac{x_{R}-x_{L}}{m+1} \cdot \frac{\partial u_{t}(x)}{\partial x}\right|_{x=\bar{x}} .
$$

We can exploit this fact to upperly bound the expression in (2), using bounds for $\left.\frac{\partial u_{t}(x)}{\partial x}\right|_{x=\bar{x}}$. Indeed, as the utility function $f$ is strictly concave, its slope is the greatest (in absolute value) when its argument is the maximum, so

$$
\max _{\theta_{t} \in \Theta}\left(\max _{x \in[0,1]} \frac{\partial u_{t}(x)}{\partial x}\right)=\left|f^{\prime}(1)\right|:=G(1) .
$$

For every $m, G(1) \frac{x_{R}-x_{L}}{m+1}$ is an upper bound of the gain that any player can get choosing $R$ instead of $L$ for any given induced outcome. Furthermore, the slope of $f$ is the lower in absolute value the smaller is its argument. Therefore, for outcomes strictly on the right of a voter's bliss point, we have

$$
\min _{x \geq \theta_{t}+\eta}\left|\frac{\partial u_{t}(x)}{\partial x}\right|=\left|f^{\prime}(\eta)\right|:=G(\eta)
$$

for every $\theta_{t} \in[0,1-\eta]$ and $\eta>0$. For every $m, G(\eta) \frac{x_{R}-x_{L}}{m+1}$ is a lower bound of the loss that a voter incurs choosing $R$ instead of $L$ when the induced outcome is on the right of her bliss point plus $\eta$.

Now, we have to determine a neighborhood of the expected vote share of party $L$ and to bound the probability that the actual vote share is in that neighborhood. By Lemma 4, indeed, the distribution of the other $m$ votes remains sufficiently close to its expected value with sufficiently high probability if $m$ is sufficiently large. Let $\eta<\delta$ and let $\varepsilon_{m}=\max \left\{0, \delta-\eta-\frac{1}{m+1}\right\}$ for every $m$. It is possible to see that, when the vote share of party $L$ induced by the other $m$ votes is not further than $\varepsilon_{m}$ from its expected value, every outcome that type $t$ can induce is on the right of $\theta_{t}+\eta \cdot{ }^{24}$ To this end, consider $\tilde{s}(m)=\tau_{L}^{*}(n)+\varepsilon_{m}$ and, with abuse of terminology, let $\hat{x}\left(L, \tau_{L}^{*}(n)+\varepsilon_{m}\right)$ denote the outcome that the voter induces choosing $L .{ }^{25} \mathrm{We}$ have

\footnotetext{
24 Of course, a necessary condition is that $m>\frac{1}{\delta-\eta}-1$ so that $\varepsilon_{m}>0$.

25 We take the largest vote share of party $L$ so that the induced outcome is the leftmost possible in the interval under consideration.
} 


$$
\begin{aligned}
\hat{x}\left(L, \tau_{L}^{*}(n)+\varepsilon_{m}\right) \\
\quad=\frac{m}{m+1}\left\{\left[\tau_{L}^{*}(n)+\varepsilon_{m}\right] x_{L}+\left[1-\tau_{L}^{*}(n)-\varepsilon_{m}\right] x_{R}\right\}+\frac{1}{m+1} x_{L} \\
\quad=\frac{m}{m+1} X\left(\tau^{*}(n)\right)-\frac{m}{m+1} \varepsilon_{m}\left(x_{R}-x_{L}\right)+\frac{1}{m+1} x_{L} \\
>X\left(\tau^{*}(n)\right)-\frac{1}{m+1}-\frac{m}{m+1} \varepsilon_{m} \\
>X\left(\tau^{*}(n)\right)-\frac{1}{m+1}-\varepsilon_{m} \\
=X\left(\tau^{*}(n)\right)-\delta+\eta \\
\geq \theta_{t}+\eta
\end{aligned}
$$

where the first inequality derives from $x_{R}-x_{L} \leq 1, x_{L} \geq 0$, and $X\left(\tau^{*}(n)\right)<1$, the second from $\frac{m}{m+1}<1$, while the last one from the assumption that $\theta_{t} \leq X\left(\tau^{*}(n)\right)-\delta$. Clearly, also the outcome that type $t$ induces choosing $R$ will be greater than $\theta_{t}+\eta$.

Thus, given expression (2) and the above discussion, we have

$$
\begin{aligned}
& U_{t}\left(R, \tau^{*}(n)\right)-U_{t}\left(L, \tau^{*}(n)\right)<\sum_{m=0}^{\infty} p(m \mid n) \\
& \quad \times\left\{\operatorname{Pr}\left(\left|\tilde{s}(m)-\tau_{L}^{*}(n)\right|>\varepsilon_{m}\right) \frac{x_{R}-x_{L}}{m+1} G(1)-\operatorname{Pr}\left(\left|\tilde{s}(m)-\tau_{L}^{*}(n)\right| \leq \varepsilon_{m}\right) \frac{x_{R}-x_{L}}{m+1} G(\eta)\right\} .
\end{aligned}
$$

Now, let

$$
\bar{\delta}=\frac{1}{2} \cdot \frac{G(\eta)}{G(1)+G(\eta)}>0 .
$$

It follows from Lemma 4 that, given $\bar{\delta}$ and $\varepsilon_{m}>0$, there exists a value $\bar{m}$ such that, for $m \geq \bar{m}, \operatorname{Pr}\left(\left|\tilde{s}(m)-\tau_{L}^{*}(n)\right|>\varepsilon_{m}\right)<\bar{\delta}$. ${ }^{26}$ Hence, the right hand side of (3) is smaller than

$$
\sum_{m=0}^{\bar{m}-1} p(m \mid n) \frac{x_{R}-x_{L}}{m+1} G(1)+\sum_{m=\bar{m}}^{\infty} p(m \mid n) \frac{x_{R}-x_{L}}{m+1}[\bar{\delta} G(1)-(1-\bar{\delta}) G(\eta)]
$$

where, for the values $m<\bar{m}$, we have used the upper bound 1 for $\operatorname{Pr}\left(\left|\tilde{s}(m)-\tau_{L}^{*}(n)\right|>\varepsilon_{m}\right)$ and the lower bound 0 for $\operatorname{Pr}\left(\left|\tilde{s}(m)-\tau_{L}^{*}(n)\right| \leq \varepsilon_{m}\right)$. Note that, given the formula of $\bar{\delta}$, we have

$$
\bar{\delta} G(1)-(1-\bar{\delta}) G(\eta)=-\frac{G(\eta)}{2}<0,
$$

so expression (4) is equivalent to

\footnotetext{
26 Differently from Lemma 4, the value of $\varepsilon_{m}$ depends on $m$. However, as $m$ increases, $\varepsilon_{m}$ becomes higher and $\operatorname{Pr}\left(\left|\tilde{s}(m)-\tau_{L}^{*}(n)\right|>\varepsilon_{m}\right)$ becomes lower. That is, if $\operatorname{Pr}\left(\left|\tilde{s}(m)-\tau_{L}^{*}(n)\right|>\varepsilon_{m}\right)<\bar{\delta}$ then, for every $m^{\prime}>m, \operatorname{Pr}\left(\left|\tilde{s}\left(m^{\prime}\right)-\tau_{L}^{*}(n)\right|>\varepsilon_{m}\right)<\bar{\delta}$ and, a fortiori, $\operatorname{Pr}\left(\left|\tilde{s}\left(m^{\prime}\right)-\tau_{L}^{*}(n)\right|>\varepsilon_{m^{\prime}}\right)<\bar{\delta}$.
} 


$$
\begin{aligned}
\sum_{m=0}^{\bar{m}-1} p(m \mid n) \frac{x_{R}-x_{L}}{m+1} G(1)-\sum_{m=\bar{m}}^{\infty} p(m \mid n) \frac{x_{R}-x_{L}}{m+1} \frac{G(\eta)}{2} \\
=\sum_{m=0}^{\bar{m}-1}\left[p(m \mid n) \frac{x_{R}-x_{L}}{m+1} G(1)-p(m+\bar{m} \mid n) \frac{x_{R}-x_{L}}{m+\bar{m}+1} \frac{G(\eta)}{2}\right] \\
\quad-\sum_{m=2 \bar{m}}^{\infty} p(m \mid n) \frac{x_{R}-x_{L}}{m+1} \frac{G(\eta)}{2} .
\end{aligned}
$$

We can finally prove that, for $n$ sufficiently large, the above expression is smaller than zero. Precisely, let

$$
\bar{n}_{L}=\left[2 \cdot \frac{(2 \bar{m}) !}{\bar{m} !} \cdot \frac{G(1)}{G(\eta)}\right]^{\frac{1}{\bar{m}}} .
$$

We can show that, for $n \geq \bar{n}_{L}$, expression (5) is negative since every term of its first summation is negative. That is, for every $m \in[0, \bar{m}-1]$,

$$
\frac{p(m \mid n)}{p(m+\bar{m} \mid n)} \cdot \frac{m+\bar{m}+1}{m+1} \leq \frac{1}{2} \cdot \frac{G(\eta)}{G(1)} .
$$

This is due to the fact that, as $n$ increases, every population realization $m$ becomes relatively less likely than the higher realization $m+\bar{m}$. In fact, note that the left hand side of (6) attains its maximum for $m=\bar{m}-1$, as

$$
\begin{aligned}
\operatorname{argmax}_{m \in[0, \bar{m}-1]} \frac{p(m \mid n)}{p(m+\bar{m} \mid n)} \cdot \frac{m+\bar{m}+1}{m+1} & =\operatorname{argmax}_{m \in[0, \bar{m}-1]} \frac{(m+\bar{m}+1) !}{(m+1) !} n^{-\bar{m}} \\
& =\bar{m}-1,
\end{aligned}
$$

and that

$$
\frac{(2 \bar{m}) !}{\bar{m} !} n^{-\bar{m}} \leq \frac{1}{2} \cdot \frac{G(\eta)}{G(1)}
$$

for $n \geq \bar{n}_{L}$.

We can conclude that, for $n \geq \bar{n}_{L}, \quad U_{t}\left(L, \tau^{*}(n)\right)>U_{t}\left(R, \tau^{*}(n)\right)$ for every $\theta_{t} \leq X\left(\tau^{*}(n)\right)-\delta$. An analogous argument implies that there exists a value $\bar{n}_{R}$ such that, for $n \geq \bar{n}_{R}, U_{t}\left(R, \tau^{*}(n)\right)>U_{t}\left(L, \tau^{*}(n)\right)$ for every $\theta_{t} \geq X\left(\tau^{*}(n)\right)+\delta$. Setting $\bar{n}=\max \left\{\bar{n}_{L}, \bar{n}_{R}\right\}$, we obtain the desired result.

For the second statement of the theorem note that, for any $n$, if $\theta^{*}(n)<\hat{\theta}$ then $X\left(\tau^{*}(n)\right)>\hat{\theta}$, since $\tau_{L}^{*}(n)=F\left(\theta^{*}(n)\right)<F(\hat{\theta})$. Hence, we have $\left|\theta^{*}(n)-X\left(\tau^{*}(n)\right)\right| \geq \max \left\{\left|\hat{\theta}-\theta^{*}(n)\right|,\left|\hat{\theta}-X\left(\tau^{*}(n)\right)\right|\right\} .^{27}$ The result directly follows.

\footnotetext{
27 The weak inequality is needed just for the case in which the points $\theta^{*}(n), X\left(\tau^{*}(n)\right)$, and $\hat{\theta}$ coincide.
} 


\section{Appendix B: Proof of Proposition 3}

Proposition 3 There exists a value $\bar{n} \in \mathbb{R}$ such that, for every $n \geq \bar{n}$, the unique Nash equilibrium is $\left(x_{L}, x_{R}\right)=(0,1)$.

Proof First, note that the assumptions on parties' preferences and on the outcome function imply that in equilibrium $x_{L}<x_{R}$. In fact, if $x_{L}=x_{R}$ then the induced outcome coincides always with the two policies, while if $x_{L} \neq x_{R}$ then the induced outcome lies between them for every voters' action profile, and every possible profile realizes with positive probability given the equilibrium of the voting game. ${ }^{28}$

Given parties' choices $x_{L}$ and $x_{R}$, let $\hat{\theta}_{x_{L}, x_{R}}$ be the associated cutpoint outcome, $\theta_{x_{L}, x_{R}}^{*}(n)$ the equilibrium cutpoint of the voting game when the expected number of voters is $n$, and $X\left(\theta_{x_{L}, x_{R}}^{*}(n)\right)$ the corresponding expected outcome, i.e. $X\left(\theta_{x_{L}, x_{R}}^{*}(n)\right)=F\left(\theta_{x_{L}, x_{R}}^{*}(n)\right) x_{L}+\left[1-F\left(\theta_{x_{L}, x_{R}}^{*}(n)\right)\right] x_{R}$. Note that, for every $x_{L}<x_{R}$, the cutpoint outcome $\hat{\theta}_{x_{L}, x_{R}}$ is strictly increasing in $x_{L}$ and in $x_{R}$. In fact, we have

$$
\frac{\partial \hat{\theta}_{x_{L}, x_{R}}}{\partial x_{L}}=\frac{F\left(\hat{\theta}_{x_{L}, x_{R}}\right)}{1+F^{\prime}\left(\hat{\theta}_{x_{L}, x_{R}}\right)\left(x_{R}-x_{L}\right)} \quad \text { and } \quad \frac{\partial \hat{\theta}_{x_{L}, x_{R}}}{\partial x_{R}}=\frac{1-F\left(\hat{\theta}_{x_{L}, x_{R}}\right)}{1+F^{\prime}\left(\hat{\theta}_{x_{L}, x_{R}}\right)\left(x_{R}-x_{L}\right)} \text {. }
$$

Both derivatives are strictly positive when $\hat{\theta}_{x_{L}, x_{R}} \notin\{0,1\}$ since $F$ is strictly increasing. By Theorem 2, we know that for every $\delta>0$ there exists a value $\bar{n} \in \mathbb{R}$ such that, for every $n \geq \bar{n}$,

$$
\left|\hat{\theta}_{x_{L}, x_{R}}-X\left(\theta_{x_{L}, x_{R}}^{*}(n)\right)\right|<\delta .
$$

Furthermore, let $\tilde{X}\left(\theta_{x_{L}, x_{R}}^{*}(n)\right)$ denote the random policy outcome given the equilibrium cutpoint $\theta_{x_{L}, x_{R}}^{*}(n)$. Given the continuity of the outcome function in the shares of votes, Lemma 5 implies that $\tilde{X}\left(\theta_{x_{L}, x_{R}}^{*}(n)\right)$ converges in probability to its expected value. That is, for every $\delta>0$ and $\varepsilon>0$ there exists a value $\bar{n} \in \mathbb{R}$ such that, for every $n \geq \bar{n}$,

$$
\operatorname{Pr}\left(\left|\tilde{X}\left(\theta_{x_{L}, x_{R}}^{*}(n)\right)-X\left(\theta_{x_{L}, x_{R}}^{*}(n)\right)\right|>\varepsilon\right)<\delta .
$$

We can now show that, for $n$ sufficiently large and for every choice $x_{R}$ of party $R$, choosing a position $x_{L}>0$ is never a best response for party $L$, as $x_{L}=0$ gives her a higher payoff. To this end, fix party $R$ 's choice $x_{R}$ and let $\bar{x}_{L}>0$. Since the cutpoint outcome is strictly increasing in $x_{L}$, we have $\hat{\theta}_{\bar{x}_{L}, x_{R}}>\hat{\theta}_{0, x_{R}}$. Let $\eta=\hat{\theta}_{\bar{x}_{L}, x_{R}}-\hat{\theta}_{0, x_{R}}$. We are going to split $\eta$ into five intervals (all equal for simplicity) and let $n$ increase. Two intervals will bound the distances between the two expected outcomes, given $\bar{x}_{L}$ and given 0 , and the corresponding cutpoint outcomes. Two other intervals will bound the distances between the two actual outcomes and the corresponding

\footnotetext{
28 As a matter of fact, the assumptions on $F$ imply that the equilibrium cutpoint of the voting game is strictly between 0 and 1 .
} 
expected outcomes. So the last interval will be the minimum distance between any two realized outcomes given the two different choices of party $L$. Formally, given the results above, there exists a value $n_{1} \in \mathbb{R}$ such that, for every $n \geq n_{1}$,

$$
\left|\hat{\theta}_{\bar{x}_{L}, x_{R}}-X\left(\theta_{\bar{x}_{L}, x_{R}}^{*}(n)\right)\right|<\frac{\eta}{5} \quad \text { and } \quad\left|\hat{\theta}_{0, x_{R}}-X\left(\theta_{0, x_{R}}^{*}(n)\right)\right|<\frac{\eta}{5} .
$$

Moreover, for every $\delta>0$ there exists a value $n_{2} \in \mathbb{R}$ such that, for every $n \geq n_{2}$,

$$
\operatorname{Pr}\left(\left|\tilde{X}\left(\theta_{\bar{x}_{L}, x_{R}}^{*}(n)\right)-X\left(\theta_{\bar{x}_{L}, x_{R}}^{*}(n)\right)\right|<\frac{\eta}{5} \cup\left|\tilde{X}\left(\theta_{0, x_{R}}^{*}(n)\right)-X\left(\theta_{0, x_{R}}^{*}(n)\right)\right|<\frac{\eta}{5}\right)>1-\delta .
$$

Let $u_{L}(x)$ be the payoff that party $L$ attains when the realized outcome is $x$, and $U_{L}\left(x_{L}, x_{R}\right)$ her expected payoff of choosing $x_{L}$ given party $R$ 's choice $x_{R}$. Let $n \geq n_{\bar{x}_{L}, x_{R}}=\max \left\{n_{1}, n_{2}\right\}$. Note that $u_{L}\left(\hat{\theta}_{0, x_{R}}+\frac{2}{5} \eta\right)-u_{L}\left(\hat{\theta}_{\bar{x}_{L}, x_{R}}-\frac{2}{5} \eta\right)=k>0$ is a lower bound of the gain that party $L$ gets choosing 0 instead of $\bar{x}_{L}$ when the outcomes realized in the two cases are no further than $\frac{2}{5} \eta$ from the corresponding cutpoints. Given the above discussion, this happens with probability greater than $1-\delta$. On the other hand, $u_{L}(1)-u_{L}(0)=h<0$ is (in absolute value) an upper bound of the loss that the party can incur in every other case, which happens with probability smaller than $\delta$. Thus, let $\delta=\frac{k}{2(k-h)}>0$. We have

$$
\begin{aligned}
U_{L}\left(0, x_{R}\right)-U_{L}\left(\bar{x}_{L}, x_{R}\right)> & (1-\delta)\left[u_{L}\left(\hat{\theta}_{0, x_{R}}+\frac{2}{5} \eta\right)-u_{L}\left(\hat{\theta}_{\bar{x}_{L}, x_{R}}-\frac{2}{5} \eta\right)\right] \\
& +\delta\left[u_{L}(1)-u_{L}(0)\right]=(1-\delta) k+\delta h=\frac{k}{2}>0,
\end{aligned}
$$

so party $L$ prefers to choose 0 rather than $\bar{x}_{L}$ given party $R$ 's choice $x_{R}$. Since this holds for every $x_{R}$ and every $\bar{x}_{L}>0$, it follows that there exists a value $\bar{n}_{L} \in \mathbb{R}$ such that, for $n \geq \bar{n}_{L}$, party $L$ has a unique best response against any choice of party $R$, that is choosing $x_{L}=0$. An analogous argument proves that there exists a value $\bar{n}_{R} \in \mathbb{R}$ such that, for $n \geq \bar{n}_{R}$, party $R$ has a unique best response against any choice of party $L$, that is choosing $x_{R}=1$. It follows that, for $n \geq \bar{n}=\max \left\{\bar{n}_{L}, \bar{n}_{R}\right\},(0,1)$ is the unique Nash equilibrium.

Acknowledgements We thank the editor and two anonymous referees for their valuable comments. We thank financial support from the Einaudi Institute for Economics and Finance and from the Italian Ministry of Education, PRIN 2017, Grant Number 2017K8ANN4.

Funding Open access funding provided by Università degli Studi di Milano within the CRUI-CARE Agreement.

Open Access This article is licensed under a Creative Commons Attribution 4.0 International License, which permits use, sharing, adaptation, distribution and reproduction in any medium or format, as long as you give appropriate credit to the original author(s) and the source, provide a link to the Creative Commons licence, and indicate if changes were made. The images or other third party material in this article are included in the article's Creative Commons licence, unless indicated otherwise in a credit line to the material. If material is not included in the article's Creative Commons licence and your intended use is not permitted by statutory regulation or exceeds the permitted use, you will need to obtain permission 
directly from the copyright holder. To view a copy of this licence, visit http://creativecommons.org/licen ses/by/4.0/.

\section{References}

Alesina A, Rosenthal H (2000) Polarized platforms and moderate policies with checks and balances. J Public Econ 75:1-20

Baron DP, Diermeier D (2001) Elections, governments, and parliaments in proportional representation systems. Q J Econ 116(3):933-967

Bouton L (2013) A theory of strategic voting in runoff elections. Am Econ Rev 103(4):1248-1288

Bouton L, Castanheira M (2012) One person, many votes: divided majority and information aggregation. Econometrica 80(1):43-87

Bouton L, Gratton G (2015) Majority runoff elections: strategic voting and Duverger's hypothesis. Theor Econ 10:283-314

Castanheira M (2003) Victory margins and the paradox of voting. Eur J Polit Econ 19(4):817-841

De Sinopoli F, Iannantuoni G (2007) A spatial voting model where proportional rule leads to two-party equilibria. Int J Game Theory 35(2):267-86

Feddersen TJ, Pesendorfer W (1999) Abstention in elections with asymmetric information and diverse preferences. Am Polit Sci Rev 93(2):381-398

Herrera H, Morelli M, Palfrey T (2014) Turnout and power sharing. Econ J 124:131-62

Jehiel P, Lamy L (2015) On discrimination in auctions with endogenous entry. Am Econ Rev 105(8):2595-2643

Krishna V, Morgan J (2011) Overcoming ideological bias in elections. J Polit Econ 119(2):183-211

Krishna V, Morgan J (2012) Voluntary voting: costs and benefits. J Econ Theory 147(6):2083-2123

Ledyard JO (1984) The pure theory of large two-candidate elections. Public Choice 44(1):7-41

Makris M (2008) Complementarities and macroeconomics: Poisson games. Games Econ Behav 62(1):180-189

Makris M (2009) Private provision of discrete public goods. Games Econ Behav 67(1):292-299

Matakos K, Troumpounis O, Xefteris D (2016) Electoral rule disproportionality and platform polarization. Am J Polit Sci 60(4):1026-1043

Meroni C (2017) Electoral competition with strategic voters. Econ Lett 160:64-66

Milgrom P, Weber R (1985) Distributional strategies for games with incomplete information. Math Oper Res 10(4):619-632

Myerson RB (1998) Population uncertainty and Poisson games. Int J Game Theory 27(3):375-392

Myerson RB (2000) Large Poisson games. J Econ Theory 94(1):7-45

Myerson RB (2002) Comparison of scoring rules in Poisson voting games. J Econ Theory 103(1):219-251

Ortuño-Ortín I (1997) A spatial model of political competition and proportional representation. Soc Choice Welf 14(3):427-438

Ritzberger K (2009) Price competition with population uncertainty. Math Soc Sci 58(2):145-157

Saporiti A (2014) Power sharing and electoral equilibrium. Econ Theory 55(3):705-729

Satterthwaite M, Shneyerov A (2007) Dynamic matching, two-sided incomplete information, and participation costs: existence and convergence to perfect competition. Econometrica 75(1):155-200

Publisher's Note Springer Nature remains neutral with regard to jurisdictional claims in published maps and institutional affiliations. 